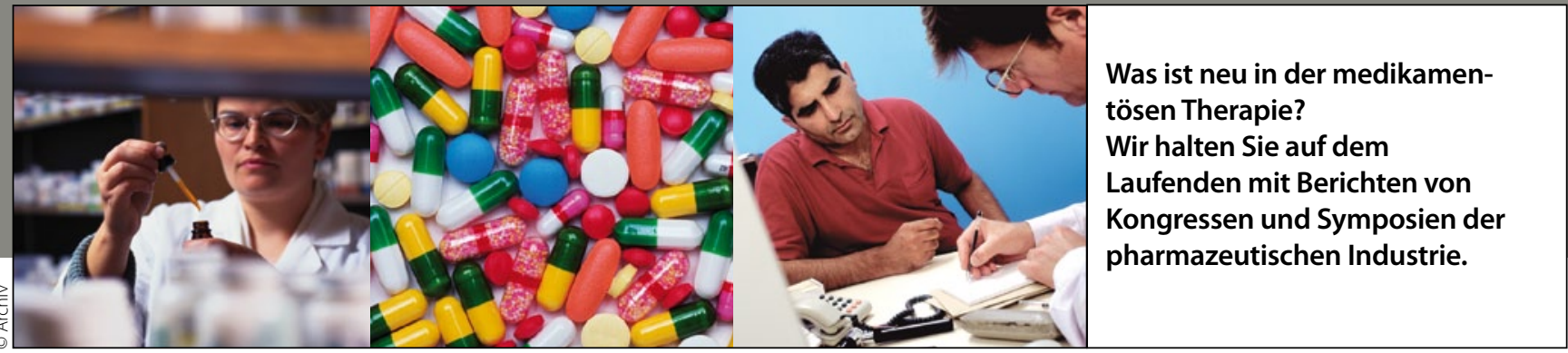

\title{
Hyperurikämie
}

\section{Deutliche Vereinfachung der Gichtdiagnostik}

- Die mikroskopische Untersuchung des Gelenkpunktats auf Uratkristalle ist aufwendig und in der hausärztlichen Routine kaum praktikabel. Einen Ausweg bietet ein simpler klinischer Score.

Aus acht Kriterien besteht der klinische Gicht-Diagnose-Score, den Ärzte aus Mexiko vergangenes Jahre vorgestellt haben. Sind die Patienten für vier oder mehr dieser Kriterien positiv, liegt mit hoher Wahrscheinlichkeit eine Gicht vor. Sieben der Kriterien sind einfach in der Praxis zu erfassen. Nur die Bestimmung des Harnsäurewerts erfordert eine Laboruntersuchung.

Fast 170 Patienten haben die Rheumatologin Dr. Janitzia Vazquez-Mellado vom Allgemeinen Krankenhaus in Mexico City und ihre Kollegen verblindet in die Studie

\section{Tabelle 1}

\section{Klinischer Gichtdiagnose- Score}

- mehr als eine Attacke einer akuten Arthritis

- Mono- oder Oligo-Arthritis-Attacken

- rasche Progression von Schmerz und Schwellung (binnen 24 Stunden)

- Podagra

- Hautrötung über dem Gelenk

_ unilaterale Tarsitis

- wahrscheinliche Tophi

- Hyperurikämie

4 oder mehr der Kriterien = Gicht einbezogen. Darunter waren 75 Patienten mit durch Punktatuntersuchung bestätigter Gicht, 30 Patienten mit rheumatoider Arthritis (RA), 31 mit Arthrose, aber auch 31 Patienten mit Spondyloarthropathien (SpA). Alle Teilnehmer wurden auf folgende Kriterien abgeklopft: mehr als eine Attacke einer akuten Arthritis, Monooder Oligo-Arthritis-Attacken, rasche Progression von Schmerz und Schwellung (binnen 24 Stunden), Podagra, Hautrötung über dem Gelenk, unilaterale Tarsitis, wahrscheinliche Tophi und Hyperurikämie.

Im Mittel erfüllten die Gichtpatienten mehr als fünf der acht Parameter, wohingegen in den anderen Patientengruppen die mittlere Zahl erfüllter Parameter bei 0,6 (Arthrose), 1,3 (RA) und 2,3 (SpA) lag. Über 90\% der Gicht-Patienten waren für vier der acht Kriterien positiv, aber nur je 3\% der RA- und Arthrose-Patienten und 6,5\% der SpA-Patienten. Die Autoren haben daraus für die Gichtdiagnose errechnet: Die Empfindlichkeit des Scores liegt bei $97 \%$ und die Genauigkeit bei $96 \%$ - wenn vier von acht Parametern positiv sind.

Auch bei speziellen Untergruppen der Gichtpatienten ermittelten die mexikanischen Kollegen eine sehr hohe Trefferquote ihres Gichtdiagnose-Scores. So waren alle Patienten mit einer Gichtmanifestation in jungen Jahren positiv in vier der acht Parameter. Weibliche Gichtpatienten wiederum erfüllten zu $94 \%$ vier der acht Parameter und bei Patienten mit sekundärer Gicht waren es $88 \%$. Der vorgestellte

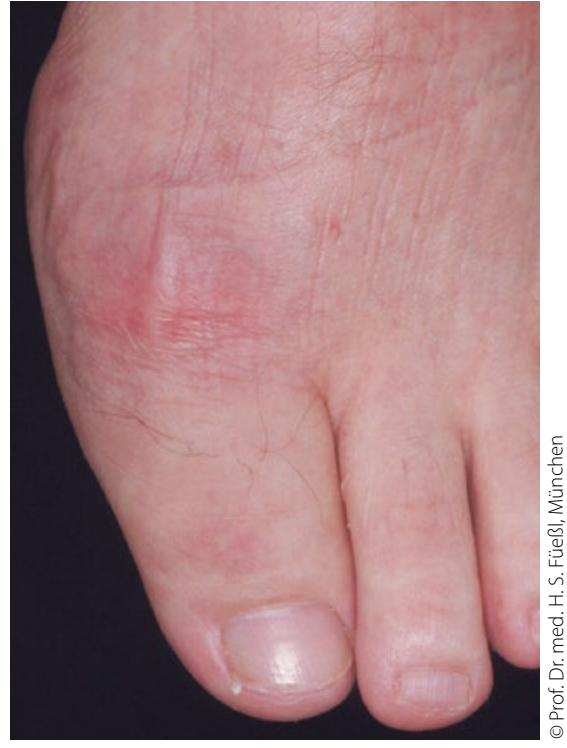

Steckt wirklich Gicht dahinter?

Gicht-Diagnose-Score ist also auch in diesen Untergruppen sehr genau. Wichtig zu wissen: Hyperurikämie und eine anamnestische Mono- oder Oligoarthritis wiesen fast $100 \%$ aller Gicht-Patienten auf.

In der Diskussion weisen die Autoren daraufhin, dass ihr Score gut mit einem anderen, schon 2010 publizierten Score übereinstimmt. In jenem Score gab es ebenfalls acht Parameter mit insgesamt 13 Punkten. Bei einem Punktwert von acht oder mehr lag zu 80\% eine Gicht vor (Arch Intern Med 2010; 170: 1120). Diesen Punktwert von acht oder mehr erfüllten $90 \%$ der Gichtpatienten aus der Studie der mexikanischen Kollegen.

- Dr. Michael Hubert

Quellen: Clin Rheumatol 2012; 31: 429 http://link.springer.com/article/10.1007/ s10067-011-1873-4;

Arch Intern Med 2010;170: 1120; http://archinte.jamanetwork.com/article.aspx? articleid $=225738$ 